\title{
Continuous evolution of risk assessment methods for cardiac surgery and intervention
}

Hoda Javadikasgari and A. Marc Gillinov

We read with great interest the thorough Review by Thalji et al. (Risk assessment methods for cardiac surgery and intervention. Nat. Rev. Cardiol. 11, 704-714; 2014). ${ }^{1}$ The authors provided an excellent overview of the development, applications, and limitations of existing cardiac surgical risk models in clinical practice. They noted limitations of these models as well as the current and future implications of these shortcomings. The validity of future risk models requires continuous evolution of today's models.

As mentioned in the Review, both clinical and administrative databases are nonstationary, and experience a phenomenon called 'concept drift.' These inherent dynamics include changes to the population of patients coming to surgery, the definition of risk factors, and the incidence of outcomes. ${ }^{3}$ An additional challenge arises because of imbalanced distribution of the outcome, particularly in small databases. ${ }^{4}$ So far, traditional cardiac surgery risk models such as EuroSCORE and the Society of Thoracic Surgeons (STS) score have been stationary models, which lose their calibration over time and, therefore, need to be reconstructed periodically from scratch (for example, EuroSCORE II) ${ }^{5,6}$

The problem of concept drift can be addressed by several methods including, but not limited to, tracking the databases over time and estimating the points at which major changes occur, ${ }^{7}$ creation of incremental models with online streaming, ${ }^{8}$ and development of ensemble models that are made up of several connected classifiers and that can autocalibrate by adding and/or deleting these classifiers over time. ${ }^{9}$ Ensemble models explicitly and simultaneously address both concept drift and imbalance phenomena. ${ }^{10}$
One of the great steps in machine learning was application of soft computing and fuzzy logic, ${ }^{11}$ which have been increasingly applied in medicine. ${ }^{12}$ Computing that can tolerate imprecision and uncertainty is termed soft computing. The potential to exploit meaningful relationships in a data set can be used in the diagnosis, treatment, and prediction of the outcome in many clinical scenarios. ${ }^{12}$ However, few studies in cardiac surgery risk assessment have utilized these possibilities. ${ }^{13,14}$

Of note, a trade-off should exist between accuracy and complexity in clinical risk assessment models. Although algorithms employing neural networks ${ }^{15}$ have not been encouraged because of their complexity, advanced algorithms exist (for example, Decision Tree) that are interpretable by both patients and experts; these algorithms have a hierarchical structure and can be used to identify nonlinear relationships between variables.

In summary, continued evolution of risk models requires new techniques to be employed. Such efforts will result in more successful models and might be particularly applicable to new procedures, such as transcatheter aortic valve implantation.

Heart and Vascular Institute, Department of Thoracic and Cardiovascular Surgery, Cleveland Clinic, 9500 Euclid Avenue, Cleveland, OH 44195, USA (H.J., A.M.G.). Correspondence to: A.M.G. gillinom@ccf.org

\section{Competing interests}

A.M.G. declares that he is a consultant for Abbott Vascular, AtriCure, ClearFlow, Edwards Lifesciences, Medtronic, On-X, St. Jude Medical, and Tendyne; and has received research support from St. Jude Medical. H.J. declares no competing interests.
1. Thalji, N. M., Suri, R. M., Greason, K. L. \& Schaff, H. V. Risk assessment methods for cardiac surgery and intervention. Nat. Rev. Cardiol. 11, 704-714 (2014).

2. Tsymbal, A. The problem of concept drift: definitions and related work [online], https:// www.cs.tcd.ie/publications/tech-reports/ reports.04/TCD-CS-2004-15.pdf (2004).

3. Hickey, G. L. et al. Dynamic prediction modeling approaches for cardiac surgery. Circ. Cardiovasc. Quality Outcomes 6, 649-658 (2013).

4. He, H. \& Garcia, E. A. Learning from imbalanced data. IEEE Trans. Knowl. Data Eng. 21, 1263-1284 (2009).

5. Nashef, S. A. et al. EuroSCORE II. Eur. J. Cardiothorac. Surg. 41, 734-744 (2012).

6. Head, S. J. et al. A systematic review of risk prediction in adult cardiac surgery: considerations for future model development. Eur. J. Cardiothorac. Surg. 43, e121-e129 (2013).

7. Alippi, C., Boracchi, G. \& Roveri, M. Just-in-time classifiers for recurrent concepts. IEEE Trans. Neural Netw. Learn. Syst. 24, 620-634 (2013).

8. Wang, S. et al. Concept drift detection for online class imbalance learning. The 2013 International Joint Conference on Neural Networks, 1-10 (2013).

9. Rokach, L. Ensemble-based classifiers. Artif. Intell. Rev. 33, 1-39 (2010).

10. Thalor, M. A. \& Patil, S. T. Review of ensemble based classification algorithms for nonstationary and imbalanced data. IOSR J. Comput. Eng. 16, 103-107 (2014).

11. Zadeh, L. A. Fuzzy sets. Informat. Control 8, 338-353 (1965).

12. Yardimci, A. Soft computing in medicine. Appl. Soft Comput. 9, 1029-1043 (2009).

13. Ghavidel, A. A. et al. Two new mathematical models for prediction of early mortality risk in coronary artery bypass graft surgery. J. Thorac. Cardiovasc. Surg. 148, 1291-1298.e1 (2014).

14. Khanmohammadi, S., Khameneh, H. S., Lewis, H. W. \& Chou, C.-A. Prediction of mortality and survival of patients after cardiac surgery using fuzzy EuroSCORE system and reliability analysis. Proced. Comput. Sci. 20, 368-373 (2013).

15. Nilsson, J. et al. Risk factor identification and mortality prediction in cardiac surgery using artificial neural networks. J. Thorac. Cardiovasc. Surg. 132, 12-19 (2006). 